OHSTPY-HEP-T-96-011

\title{
The Vacuum in Light-Cone Field Theory
}

\author{
David G. Robertson \\ Department of Physics, The Ohio State University, Columbus, OH 43210
}

(October 8, 2018)

\begin{abstract}
This is an overview of the problem of the vacuum in light-cone field theory, stressing its close connection to other puzzles regarding light-cone quantization. I explain the sense in which the light-cone vacuum is "trivial," and describe a way of setting up a quantum field theory on null planes so that it is equivalent to the usual equal-time formulation. This construction is quite helpful in resolving the puzzling aspects of the light-cone formalism. It furthermore allows the extraction of effective Hamiltonians that incorporate vacuum physics, but that act in a Hilbert space in which the vacuum state is simple. The discussion is fairly informal, and focuses mainly on the conceptual issues. Additional technical details of the construction described here will appear in a forthcoming paper written with Kent Hornbostel.
\end{abstract}

Talk presented at Orbis Scientiae 1996, Miami Beach, FL, January 25-28, 1996. To appear in the proceedings. 


\section{INTRODUCTION}

The most striking aspect of light-cone field theories is surely the claim that the vacuum state is simple, or even trivial. The basic argument for this is elementary. We begins by noting that the longitudinal momentum $P^{+}=P^{0}+P^{3}$ is positive semidefinite and conserved in interactions. However, from the free-particle dispersion relation in light-cone coordinates,

$$
P^{-}=\frac{P_{\perp}^{2}+m^{2}}{P^{+}}
$$

it follows that the only finite-energy states other than the bare vacuum that have $P^{+}=0$ contain massless quanta with $P_{\perp}=0$ - a set of measure zero in more than $1+1$ dimensions. Thus if these can be ignored as unimportant in the full theory, then the bare vacuum is the only state in the theory with $P^{+}=0$, and so must be an exact eigenstate of the full interacting Hamiltonian.

If true, this enormously simplifies any effort to solve field theories nonperturbatively using Hamiltonian diagonalization, as attempts to compute the spectrum and wavefunctions of physical states are not complicated by the need to recreate a ground state in which processes occur at unrelated locations and energy scales. It furthermore results in a constituent picture, in which all quanta in, say, a hadron's wavefunction are specifically related to that hadron. This allows for an unambiguous definition of the partonic content of hadrons, and makes interpretation of the wavefunctions straightforward. For this reason the light-cone framework is the most natural one in which to encode hadronic properties [面].

It immediately raises the question, however, of whether field theories quantized on the light cone are equivalent in all respects with the corresponding equal-time theories. In many cases, notably QCD, there is important physics that in the usual language is attributed to the properties of the vacuum. The fact that the pion is light on the hadronic scale is conventionally traced to spontaneous breaking of the axial flavor symmetries. It is not clear how a trivial vacuum can be a spontaneously broken vacuum of anything. Furthermore, the QCD ground state is a superposition of "topological" vacua labeled by a parameter $\theta$. This structure is important for understanding why there is no ninth light pseudoscalar meson (for three light flavors), and for satisfying cluster decomposition. In addition, if $\theta \neq 0$ (and none of the quarks are massless), then QCD violates $P$ and $T$, leading for example to a nonzero electric dipole moment for the neutron. The structure of the vacuum in QCD is clearly connected to observable consequences of the theory.

One way of reconciling the apparent triviality of the vacuum in light-cone quantization with the fact that the vacuum has nontrivial physical consequences is based on the following set of observations [2]. Eqn. (1.1) implies that particle states that can mix with the bare vacuum are high-energy states. This suggests that it is natural to think in terms of effective Hamiltonians. That is, we may imagine introducing an explicit cutoff on longitudinal momentum for particles

$$
P^{+}>\lambda
$$

(For practical calculations it may be preferable to implement the cutoff in some more sophisticated way. I use this simple scheme to illustrate the point.) This immediately makes the vacuum trivial and gives the resulting constituent picture. In principle, it should then 
be possible to restore the effects of states with $P^{+}<\lambda$, including the physics of the vacuum, by means of effective interactions which, because the states eliminated have large light-cone energies, will be local in $x^{+}$. From this point of view, the statement of the vacuum problem on the light cone is: How can we compute these effective interactions?

I should perhaps emphasize at this point that the physical vacuum state in light-cone field theory is not really trivial. It cannot be, if the light-cone theory is to be equivalent to the corresponding equal-time theory. The potential advantage of the light-cone approach is that it may allow us to move the effects of the vacuum into the Hamiltonian and work with a simple vacuum state. The problem is that sorting out the structure of the vacuum in light-cone quantization is especially difficult. At the kinematical point at which the vacuum lives there is a diverging density of states with diverging energies, but for which matrix elements of the Hamiltonian also are singular. This is the loophole in the simple argument for vacuum triviality: states with $P^{+}=0$ do have divergent energies, but they are infinitely strongly coupled to low-energy states. It therefore does not follow that they decouple from low-energy physics.

Some sort of small- $P^{+}$regularization is necessary even without the motivation of obtaining a constituent picture, and how we approach the problem of computing the effective interactions will certainly depend on the specific choice of this regulator. For example, if we think in terms of simple momentum cutoffs then a renormalization group approach is natural [3] (the effective interactions are the counterterms that remove dependence on the cutoff $\lambda$ from physical quantities). Another method that has been studied in some detail involves using discretization as an infrared regulator 国. In this case any vacuum structure must be connected with the properties of the zero modes (in the Fourier sense) of the fields.

Both of these cutoffs, however, force the vacuum to be trivial from the outset, and we face the problem of how to put the vacuum physics back in. The RG approach should work in principle - for a particular set of cutoffs there is presumably a single effective Hamiltonian that gives completely cutoff-independent results and corresponds to the QCD Hamiltonian. The difficulty here is a practical one. It is not clear whether this program can really be carried out, particularly since the use of perturbation theory to construct the RG is probably not sufficient for QCD. The development of nonperturbative RGs for use in light-cone field theory is an extremely interesting and challenging problem.

Discretized light-cone quantization (DLCQ) is a self-contained formalism with the inclusion of the zero modes, so the question here is simply whether the vacuum physics is present or not at the end of the day. Analysis of simple models suggests that certain mean-field aspects of the vacuum are in fact captured by the zero modes [5]; however, not all of the relevant structure is present. In the $\phi_{1+1}^{4}$ model, for example, the zero mode gives rise to the expected strong-coupling phase transition, but with the (incorrect) mean-field critical exponent. This suggests that even with zero modes DLCQ does not contain all of the necessary physics.

The main purpose of this talk is to describe one way of regulating light-cone field theories

\footnotetext{
${ }^{1}$ It is instructive to contrast this with the analogous procedure in equal-time quantization. In this case there are states that are kinematically allowed to mix with the vacuum at all energy scales, so that a description of vacuum physics in terms of an effective Hamiltonian is not practicable.
} 
that does not force the vacuum to be trivial from the beginning. In fact, the construction is designed to be completely equivalent to the usual equal-time formalism, so that there is no confusion about what is or is not present in the theory. I shall also sketch how it can be used to extract effective light-cone Hamiltonians, which incorporate the physical effects of the vacuum but act in a space with a trivial ground state.

In order to widen the context of the discussion, however, let me mention a few other "classic" puzzles regarding light-cone quantization. All of these problems, including that of the vacuum, are interrelated, and all of them are clarified by the construction I shall describe.

- It has been noted on occasion that a null plane is not a good Cauchy surface for a relativistic wave equation; in fact, it is a surface of characteristics for such an equation [6]. Thus initial conditions on one such surface plus a Hamiltonian are not in general sufficient to determine a general solution to the field equations. In the quantum field theory this problem is manifested as missing degrees of freedom. A particularly clear example of this is that of a free massless fermion in two spacetime dimensions, as first discussed by McCartor [7]. Here we find, following the usual light-cone procedure, that the theory contains only right-moving particles; half of the solution space is missing. Additional degrees of freedom, initialized on a second null plane, are required to properly incorporate the left-movers. One may have intuitively the idea that this is a problem specific to massless fields in $1+1$ dimensions (or for only those massless particles with $p_{\perp}=0$ in higher dimensions - a set of measure zero), but I shall argue that this is not the case.

- It is not clear what, if any, boundary conditions can be consistently imposed on a null plane. The problem is that some points on the surface are causally connected, and requiring fields at such points to be related in some way may result in an overdetermined system. In other words, some boundary conditions may be in conflict with the dynamics we wish to solve.

- There seems to be a mysterious discontinuity in the way massless and massive fields are treated on the light cone. As mentioned above, for a free massless fermion in $1+1$ dimensions we must include independent degrees of freedom on two different null planes to recover the correct theory. For nonzero mass, however, this does not seem to be necessary; the usual light-cone construction gives a theory that is precisely isomorphic to the equal-time theory of a free massive Fermi field. Thus the limit is apparently not smooth - it seems that additional degrees of freedom must be switched on precisely at $m=0$. We shall argue below that this apparent discontinuity is an artifact of considering only free field theories. In an interacting theory a small- $k^{+}$ regulator must be employed, and this allows for a completely unified treatment of massive and massless fields. In particular, the massless limit may be taken smoothly.

- Finally let us mention the "No-problem" problem. This is that simply ignoring most or all of these problems sometimes works. For example, the spectrum of the Schwinger model can be computed rather well without worrying about any of the above [8]. This is somewhat astonishing considering that this is a situation where these problems might be expected to be at their most severe. All the fields are massless, the free fermion 


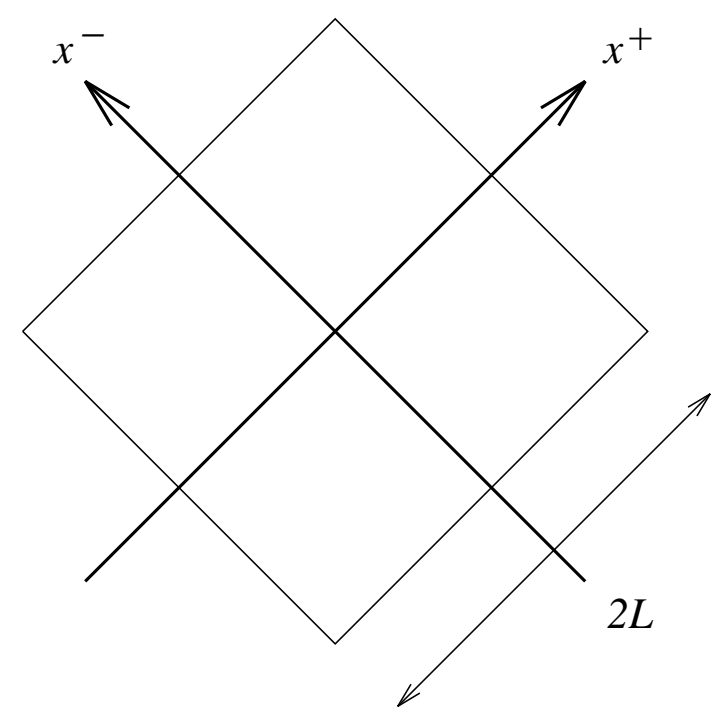

FIG. 1. Finite volume used for infrared regulation.

theory is known to be missing half of the necessary states, and all points on the initial-value surface are in causal contact. There are many aspects of the Schwinger model that simply cannot be understood without worrying about these problemsthe anomaly, the occurrence of a condensate, the $\theta$-vacuum [9]-but the spectrum of physical bosons is reproduced exactly. It would be nice if this feature were to hold in more complicated examples, that certain things would be computable even without a complete formulation incorporating all the subtleties. One of the goals of the work I will describe is to try to understand this in a quantitative way, by starting from a manifestly complete formalism so that the effects of discarding certain pieces of physics can be studied without ambiguity.

The rest of this paper is organized as follows. In the next section I discuss infrared regularization using a finite volume, and the initial-value problem in such a box. I show that with a careful treatment of the boundary surfaces this formulation is completely equivalent to equal-time quantization. In particular, all conserved charges (including the Hamiltonian) are guaranteed to be identical to the operators we would construct in equal-time quantization, and the vacuum state is complicated. I then describe the formalism in detail for scalar and Fermi fields. Finally, I sketch how to use this construction to obtain effective Hamiltonians for use in light-cone field theories with a simple vacuum. 


\section{LIGHT-CONE INITIAL VALUE PROBLEM IN A FINITE VOLUME}

As discussed above, one aspect of the vacuum problem on the light cone is that it is difficult to find an infrared regulator that does not automatically remove the vacuum structure. One way of achieving this involves using a finite volume as a regulator and treating the initial-value problem carefully [0,10,11]. This analysis will show that extra degrees of freedom, beyond what is present in the conventional light-cone quantization, are required to construct a theory that is equivalent to the equal-time theory. These additional degrees of freedom are what will allow complicated vacuum structure to occur.

To be more concrete, let us consider a free massive scalar field in $1+1$ dimensions confined to the region shown in Fig. 1. (The extension to higher dimensions is straightforward and is discussed in [11].) The first question is: What classical data are required in order to determine a completely general solution to the field equation

$$
\left(4 \partial_{+} \partial_{-}+m^{2}\right) \phi=0 ?
$$

These data will correspond to independent operators in the quantum field theory.

Let us begin by assuming that we have specified the value of $\phi$ on the conventional lightcone initial-value surface $x^{+}=0$, for $-L \leq x^{-} \leq L$. Eqn. (2.1) then allows us to evolve $\partial_{-} \phi$ infinitesimally in $x^{+}$:

$$
\partial_{-} \phi\left(\epsilon, x^{-}\right)=\partial_{-} \phi\left(0, x^{-}\right)+\epsilon \partial_{+}\left(\partial_{-} \phi\left(0, x^{-}\right)\right)
$$

where

$$
\partial_{+}\left(\partial_{-} \phi\right)=-\frac{m^{2}}{4} \phi
$$

In order to go on, however, we need $\phi$ itself on the new $x^{+}$-slice. This is obtained by integration,

$$
\phi\left(\epsilon, x^{-}\right)=\int_{-L}^{x^{-}} d y^{-} \partial_{-} \phi\left(\epsilon, y^{-}\right)+\phi(\epsilon,-L)
$$

which of course requires knowing the value of the field at one point on the surface; here we have arbitrarily taken this point to be $(\epsilon,-L)$. Now that we have $\phi$ at $x^{+}=\epsilon$, we can continue to the next slice, and so on. Clearly the process can be iterated to fill out the entire box, provided that in addition to knowing $\phi$ on $x^{+}=0$, we are also given $\phi$ along a surface of constant $x^{-}$.

We thus find that in order to obtain a general solution to Eqn. (2.1) we must specify $\phi$ both at $x^{+}=0$ (or more generally on any surface of constant $x^{+}$) and on a surface of constant $x^{-}$. This means that when we build the quantum field theory we should include independent operators initialized on some surface of constant $x^{-}$that represent this classical freedom. This simple analysis does not tell us which surfaces of constant $x^{-}$are most useful, however. In order to answer this question it is helpful to think about actually setting up the quantum theory with fields initialized on both surfaces. The basic problem is to write down expressions for the fields and find a set of commutation relations among them such that, e.g., the Poincaré generators satisfy the correct algebra and generate the proper transformations 


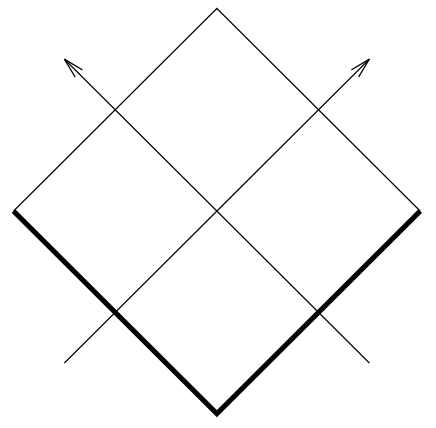

(a)

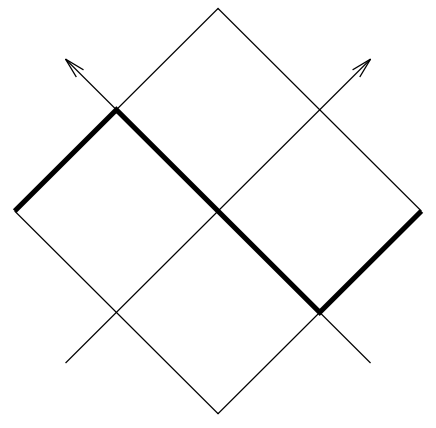

(b)

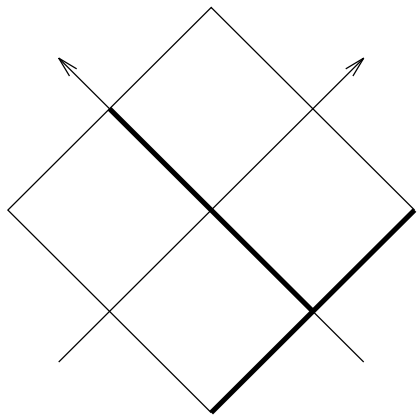

(c)

FIG. 2. Some possible initial-value surfaces. The dark lines indicate where initial data are specified.

on the fields. One important point is that in all but the simplest cases (e.g., free field theories), the commutation relations between field operators at time-like separations are a priori unknown. We should therefore only consider surfaces that contain no time-like separated points. Two particularly useful surfaces of this type are shown in Figs. 2(a) and (b). The surface shown in Fig. 2(c) is not suitable.

The two "good" surfaces each have particular advantages. The symmetric one [Fig. 2(a)] is manifestly parity invariant, for example. (Recall that under parity $x^{+} \leftrightarrow x^{-}$, so that the two wings are interchanged.) This is helpful because initializing the fields is completely symmetric on the two wings. The asymmetric surface [Fig. 2(b)] will be more useful, however, when we attempt to extract an effective Hamiltonian for use in light-cone theories of the usual type, that is, involving the fields initialized at $x^{+}=0$.

In fact, the fields on the asymmetric surface can be obtained from those on the symmetric one by means of a shift in $x^{+}$and a minor reorganization of the degrees of freedom [11]. I shall therefore concentrate first on the symmetric surface, where initialization is easier, and shift to obtain the formulation on the asymmetric surface only when we are ready to discuss effective Hamiltonians.

\section{A. Conserved Charges}

How do these additional degrees of freedom enter the dynamics? That is, how are they incorporated into the Hamiltonian and other conserved charges of the theory? As we shall see, understanding this leads to certain clarifications regarding the equivalence of the lightcone and equal-time formalisms.

The basic point is quite simple [7]. For any conserved current,

$$
\partial_{\mu} J^{\mu}=0
$$

we have

$$
\oint d \sigma_{\mu} J^{\mu}=0
$$

where the integral is taken over a closed surface. If this is taken to be the surface shown in Fig. 3, for example, then Eqn. (2.6) implies 


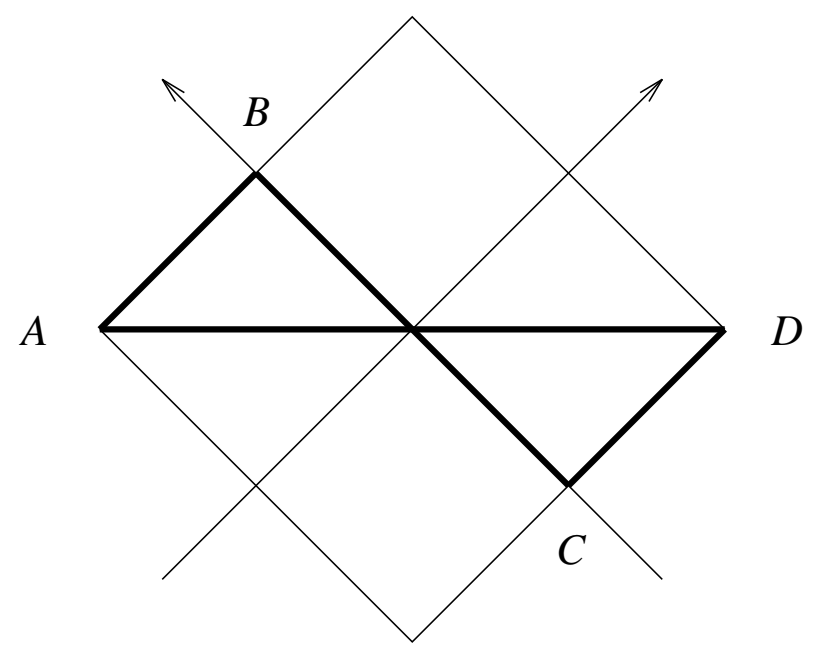

FIG. 3. Integration contour for conserved charges.

$$
\begin{aligned}
Q & =\int_{A}^{D} d x J^{0} \\
& =\frac{1}{2} \int_{A}^{B} d x^{+} J^{-}+\frac{1}{2} \int_{C}^{B} d x^{-} J^{+}+\frac{1}{2} \int_{C}^{D} d x^{+} J^{-} .
\end{aligned}
$$

(The factors of $1 / 2$ are due to our definition of light-cone coordinates.) The middle term in the second line is the usual expression for the charge in light-cone quantization. We therefore see that inclusion of contributions from the boundaries is in general necessary if the various charges are to be identical to those we would construct in equal-time quantization.

To see why the vacuum is complicated when the boundaries are retained, consider the particular case of the energy-momentum tensor. We have, for example,

$$
P^{+}=\frac{1}{2} \int_{A}^{B} d x^{+} T^{-+}+\frac{1}{2} \int_{C}^{B} d x^{-} T^{+-}+\frac{1}{2} \int_{C}^{D} d x^{+} T^{-+} .
$$

The boundary contributions involve $T^{-+}$, which contains interaction terms. Thus $P^{+}$will no longer be diagonal in a Fock basis, and the physical vacuum state can be complicated.

Recognizing the role played by the boundaries is crucial in resolving certain specific puzzles of light-cone quantization. For example, in the Schwinger model (electrodynamics of massless fermions in $1+1$ dimensions) the vector and axial-vector currents are related by

$$
\begin{aligned}
& J_{5}^{+}=J^{+} \\
& J_{5}^{-}=-J^{-} .
\end{aligned}
$$

In the usual light-cone formalism, where charges are obtained by integrating only over $x^{+}=$ 0 , this implies

$$
Q=\frac{1}{2} \int d x^{-} J^{+}=Q_{5}
$$

But $Q_{5}$ is supposed to be anomalous, while $Q$ is conserved. The resolution of this paradox is that contributions from the boundary surfaces also must be included. These involve integrals of $J_{(5)}^{-}$over a surface of constant $x^{-}$, so that a difference between $Q$ and $Q_{5}$ can arise [9]. 
It might be thought that this sort of problem arises only for massless fields in $1+1$ dimensions, or for the $p_{\perp}=0$ modes (only) of a massless field in higher dimensions. This is incorrect, however, and is a result of considering only free field theories. In a free massive theory the limit of large box size can trivially be taken, since there are no divergent matrix elements of the Hamiltonian.'] Thus the boundary fields decouple completely and what remains is the usual light-cone theory. This does not happen for a massless field (or for the $p_{\perp}=0$ modes of a higher-dimensional theory); in this case the left- and right-moving modes are not coupled and so they can never decouple. This is what leads to the apparent paradox regarding the difference between massless and massive fields on the light cone, and is a point I would like particularly to stress. The need to include boundary fields in order to obtain a light-cone formulation that is equivalent to the equal-time theory is quite general, and applies to both massive and massless fields in any number of dimensions. What leads to confusion is that in free field theory the boundaries can be decoupled for massive fields. (This is because the vacuum really is trivial for free field theories!) In an interacting theory, however, the infinite-volume limit need not be trivial, and singularities in the interactions can lead to observable consequences - vacuum condensates, for example - that would simply be absent if the theory were formulated without the boundary degrees of freedom.

In the next two sections I will show how to realize this scheme in some simple cases. The central problem is to obtain general expressions for the fields on the initial surfaces such that all requirements of relativistic quantum field theory, in particular causality, are satisfied, and no boundary conditions are employed that are in conflict with the dynamics. The discussion will necessarily be rather incomplete; my goal is mainly to give a flavor of how the formalism is set up. Further examples and many additional details are given in [11].

\section{SCALARS}

Let us begin by considering a free, massless scalar field, quantized on the symmetric surface I. This is a very simple example, but it serves to illustrate many of the basic points.

It is convenient to impose periodic boundary conditions at equal time,

$$
\phi(-L, L)=\phi(L,-L) .
$$

This insures that the Hamiltonian and other conserved charges are time-independent. Note that because the two points thus related are separated by a space-like interval, there is no conflict with causality. The equation of motion is simply

\footnotetext{
${ }^{2}$ More precisely, there is no divergent coupling between states. The free energies diverge for $p^{+} \rightarrow 0$, but we can argue that this is irrelevant, as the states for which this occurs are not in the spectrum of either the light-cone or equal-time theories - they have infinite energy.

${ }^{3}$ This theory does not actually exist except with an infrared regulator such as the box in place. The fact that massless elementary scalars are afflicted with unmanageable infrared divergences is the essence of Coleman's theorem regarding the impossibility of spontaneous breaking of continuous symmetries in two spacetime dimensions; the necessary Goldstone boson cannot exist. Here we shall never try to remove the regulator, so that the theory is always well defined.
} 


$$
\partial_{+} \partial_{-} \phi=0
$$

which has as its general solution

$$
\phi\left(x^{+}, x^{-}\right)=\phi_{+}\left(x^{+}\right)+\phi_{-}\left(x^{-}\right)
$$

where $\phi_{ \pm}$are arbitrary functions. It is clear that initial data on both $x^{+}=-L$ and $x^{-}=-L$ are required to obtain the full solution space of the theory. Our first problem is to find the most general expression for the fields consistent with, e.g., causality, the equations of motion, and the condition (3.1).

Now the equation of motion itself tells us what boundary conditions are allowed on the light-cone surfaces. To see this, integrate it over one or the other of the wings. We obtain, for example,

$$
\partial_{-} \phi(L,-L)=\partial_{-} \phi(-L,-L)
$$

which implies, because of the equal-time periodicity condition,

$$
\partial_{-} \phi(-L, L)=\partial_{-} \phi(-L,-L) \text {. }
$$

Thus the derivative $\partial_{-} \phi$ may be taken to be periodic on $x^{+}=-L$. Similarly, $\partial_{+} \phi$ may be taken to be periodic on the surface $x^{-}=-L$. We can therefore write general Fourier expansions for these,

$$
\begin{aligned}
& \partial_{-} \phi\left(-L, x^{-}\right)=\partial_{-} \varphi\left(x^{-}\right)+\frac{\phi_{0}}{L} \\
& \partial_{+} \phi\left(x^{+},-L\right)=\partial_{+} \widetilde{\varphi}\left(x^{+}\right)+\frac{\widetilde{\phi}_{0}}{L},
\end{aligned}
$$

where $\varphi$ and $\tilde{\varphi}$ are sums of periodic oscillators and $\phi_{0}$ and $\widetilde{\phi}_{0}$ are the zero-momentum modes. (The factors of $L$ are introduced for later convenience.) Explicitly,

$$
\begin{aligned}
& \varphi\left(x^{-}\right)=\frac{1}{\sqrt{2 \pi}} \sum_{q=2,4, \ldots} \frac{1}{\sqrt{q}}\left(a_{q} e^{-i q \pi x^{-} / 2 L}+a_{q}^{\dagger} e^{+i q \pi x^{-} / 2 L}\right) \\
& \widetilde{\varphi}\left(x^{+}\right)=\frac{1}{\sqrt{2 \pi}} \sum_{q=2,4, \ldots} \frac{1}{\sqrt{q}}\left(\widetilde{a}_{q} e^{-i q \pi x^{+} / 2 L}+\widetilde{a}_{q}^{\dagger} e^{+i q \pi x^{+} / 2 L}\right) .
\end{aligned}
$$

We can then integrate Eqns. 3.6)-(3.7) to obtain the fields on the initial value surfaces, in terms of the given derivatives and the value of the field at the corner point $(-L,-L)$. We obtain

$$
\begin{aligned}
& \phi\left(-L, x^{-}\right)=\varphi\left(x^{-}\right)-\varphi(-L)+\frac{\phi_{0}}{L}\left(x^{-}+L\right)+\phi(-L,-L) \\
& \phi\left(x^{+},-L\right)=\widetilde{\varphi}\left(x^{+}\right)-\widetilde{\varphi}(-L)+\frac{\widetilde{\phi}_{0}}{L}\left(x^{+}+L\right)+\phi(-L,-L) .
\end{aligned}
$$

Imposing the condition (3.1) then leads to the identification 


$$
\phi_{0}=\widetilde{\phi}_{0}
$$

Finally, it is convenient to define

$$
\phi(-L,-L) \equiv \varphi(-L)+\widetilde{\varphi}(-L)+\psi
$$

so that

$$
\begin{aligned}
\phi\left(-L, x^{-}\right) & =\varphi\left(x^{-}\right)+\widetilde{\varphi}(L)+\frac{\phi_{0}}{L}\left(x^{-}+L\right)+\psi \\
\phi\left(x^{+},-L\right) & =\widetilde{\varphi}\left(x^{+}\right)+\varphi(L)+\frac{\phi_{0}}{L}\left(x^{+}+L\right)+\psi .
\end{aligned}
$$

These represent the most general expressions for the fields on the initial surfaces that are consistent with the equations of motion and the periodicity condition (3.1). The classical initial data are the Fourier modes $a_{q}$ and $\widetilde{a}_{q}$, and the numbers $\phi_{0}$ and $\psi$. In the quantum theory these become operators whose commutation relations are to be determined by demanding that the correct Heisenberg equations and Poincaré algebra are obtained.t

Note that the fields are not themselves periodic on either of the surfaces. Imposing periodicity on the fields, as one would do in DLCQ, corresponds to a less general expression for the fields, or, equivalently, to focusing on a subset of the classical solution space. In the quantum theory this corresponds to omitting degrees of freedom.

The Poincaré generators receive contributions from both wings of the initial-value surface:

$$
P^{\mu}=\frac{1}{2} \int_{-L}^{L} d x^{+} T^{-\mu}\left(x^{+},-L\right)+\frac{1}{2} \int_{-L}^{L} d x^{-} T^{+\mu}\left(-L, x^{-}\right) .
$$

The only nonvanishing components of the energy-momentum tensor are

$$
\begin{aligned}
& T^{++}=4\left(\partial_{-} \phi\right)^{2} \\
& T^{--}=4\left(\partial_{+} \phi\right)^{2}
\end{aligned}
$$

so that

$$
\begin{aligned}
& P^{+}=2 \int_{-L}^{L} d x^{-}\left[\partial_{-} \phi\left(-L, x^{-}\right)\right]^{2} \\
& P^{-}=2 \int_{-L}^{L} d x^{+}\left[\partial_{+} \phi\left(x^{+},-L\right)\right]^{2} .
\end{aligned}
$$

These are guaranteed to be the same operators, though expressed in a different representation, as we would obtain in an equal-time quantization of this theory. Requiring that the Heisenberg equations reproduce the field equation (3.2) leads to the commutation relations

\footnotetext{
${ }^{4}$ Note that the regulator breaks longitudinal boost invariance, so that the commutator $\left[P^{ \pm}, K\right]=$ $\mp 2 i P^{ \pm}$is not recovered for finite $L$. In practice the Heisenberg equations are usually sufficient to determine the field algebra, perhaps supplemented by the requirement that $\left[P^{+}, P^{-}\right]=0$ and that the fields commute for space-like separations.
} 


$$
\begin{aligned}
{\left[\varphi\left(x^{-}\right), \varphi\left(y^{-}\right)\right] } & =-\frac{i}{4} \epsilon\left(x^{-}-y^{-}\right)+\frac{i}{4 L}\left(x^{-}-y^{-}\right) \\
{\left[\widetilde{\varphi}\left(x^{+}\right), \widetilde{\varphi}\left(y^{+}\right)\right] } & =-\frac{i}{4} \epsilon\left(x^{+}-y^{+}\right)+\frac{i}{4 L}\left(x^{+}-y^{+}\right) \\
{\left[\psi, \phi_{0}\right] } & =\frac{i}{4}
\end{aligned}
$$

where $\epsilon$ is the antisymmetric step function. In terms of the Fock operators, the first two commutators correspond to

$$
\left[a_{q}, a_{p}^{\dagger}\right]=\left[\widetilde{a}_{q}, \widetilde{a}_{p}^{\dagger}\right]=\delta_{q, p} .
$$

Furthermore, with the redefinitions

$$
\begin{aligned}
\psi & \equiv \frac{1}{2 \sqrt{2}}\left(b+b^{\dagger}\right) \\
\phi_{0} & \equiv \frac{i}{2 \sqrt{2}}\left(b^{\dagger}-b\right),
\end{aligned}
$$

Eqn. (3.23) can be recast as $\left[b, b^{\dagger}\right]=1$.

With these commutation relations the Poincaré generators correctly translate the fields in $x^{ \pm}$, and furthermore satisfy, $\left[P^{+}, P^{-}\right]=0$. We thus have a satisfactory representation of the theory, including all the relevant degrees of freedom. The Fock space is spanned by states of the form

$$
a_{p_{1}}^{\dagger} \ldots a_{p_{n}}^{\dagger} \widetilde{a}_{q_{1}}^{\dagger} \ldots \widetilde{a}_{q_{m}}^{\dagger}\left(b^{\dagger}\right)^{l}|0\rangle
$$

where $|0\rangle$ is annihilated by $a_{q}, \widetilde{a}_{q}$, and $b$. The Poincaré generators are given by

$$
\begin{aligned}
& P^{-}=\sum_{q}\left(\frac{q \pi}{L}\right) \widetilde{a}_{q}^{\dagger} \widetilde{a}_{q}+\frac{4}{L}\left(\phi_{0}\right)^{2} \\
& P^{+}=\sum_{q}\left(\frac{q \pi}{L}\right) a_{q}^{\dagger} a_{q}+\frac{4}{L}\left(\phi_{0}\right)^{2} .
\end{aligned}
$$

In fact, the entire construction can be shown to be correct by mapping it onto the equaltime theory of a massless scalar in a box. That is, we can quantize the theory at equal time with the periodicity condition (3.1), and solve the resulting theory to obtain the fields on the boundary surfaces. We then identify the light-cone Fock operators in terms of the equal-time ones, and so obtain the relation between the two representations. Not surprisingly, the $a_{q}$ modes correspond to right-moving quanta, the $\widetilde{a}_{q}$ to left-moving quanta, and $\phi_{0}$ to the zeromomentum mode in the equal-time Fourier expansion. The Hamiltonian and momentum operators can also be constructed and are identical to the appropriate combinations of (3.28) and (3.29).

The massive case is more involved, essentially because the left and right movers are coupled by the mass term (for example, a finite boost can now change a left mover to a right mover, and vice versa). The commutator we wish to reproduce in this case is the Pauli-Jordan function 


$$
[\phi(x), \phi(y)]=-\frac{i}{4}\left[\epsilon\left(x^{+}-y^{+}\right)+\epsilon\left(x^{-}-y^{-}\right)\right] J_{0}\left(\mu \sqrt{(x-y)^{2}}\right)
$$

where $\mu$ is the mass. This is properly causal; it vanishes whenever $(x-y)$ is space-like. Furthermore, the Bessel function is unity on the light cone, reproducing the usual lightcone commutator of the fields. Light-cone commutators involving derivatives of the field, however, can be $\mu$-dependent. For example,

$$
\left[\partial_{-} \phi(-L,-L), \partial_{+} \phi\left(x^{+},-L\right)\right]=\frac{i \mu^{2}}{16} \text {. }
$$

This suggests that $\mu$ should be built in to the expressions for the fields, in such a way that those commutators involving derivatives that are needed are correctly reproduced.

This can in fact be done, and the details are given in [11. For now, however, let us turn to the case of a massive Fermi field. This is similar in many ways due to the presence of the mass, but allows for a slightly less cluttered treatment.

\section{FERMIONS}

Consider a free, massive Dirac fermion in $1+1$ dimensions, quantizing again on the symmetric surface. The standard light-cone decomposition of a Fermi field,

$$
\psi_{ \pm}=\frac{1}{2} \gamma^{0} \gamma^{ \pm} \psi
$$

allows the separation of the Dirac equation into the coupled pair

$$
\begin{aligned}
& i \partial_{+} \psi_{+}=\frac{m}{2} \psi_{-} \\
& i \partial_{-} \psi_{-}=\frac{m}{2} \psi_{+} .
\end{aligned}
$$

A classical analysis of these, analogous to that given in Sect. II for the scalar field, indicates that in order to determine their general solution we must specify $\psi_{+}$on $x^{+}=-L$ and $\psi_{-}$on $x^{-}=-L$. The problem is thus to find expressions for the quantum fields on these surfaces that furnish a representation of the free-field canonical anticommutation relations, are properly causal, etc. The relevant commutators are

$$
\begin{aligned}
& \left\{\psi_{+}\left(-L, x^{-}\right), \psi_{+}^{\dagger}\left(-L, y^{-}\right)\right\}=\delta\left(x^{-}-y^{-}\right) \\
& \left\{\psi_{-}\left(x^{+},-L\right), \psi_{-}^{\dagger}\left(x^{+},-L\right)\right\}=\delta\left(x^{+}-y^{+}\right) \\
& \left\{\psi_{+}\left(-L, x^{-}\right), \psi_{-}^{\dagger}\left(-L, y^{-}\right)\right\}=-\frac{i m}{4} \epsilon\left(x^{-}-y^{-}\right) .
\end{aligned}
$$

In addition, all field operators should anticommute for space-like separations.

For $m=0$ the necessary construction has been given in Ref. [7]. After imposing antiperiodicity at equal time,

$$
\psi_{ \pm}(-L, L)=-\psi_{ \pm}(L,-L)
$$


an argument identical to that given for the massless scalar shows that $\psi_{+}$may be chosen to be antiperiodic on $x^{+}=-L$ and $\psi_{-}$may be chosen to be antiperiodic on $x^{-}=-L$. Thus we may write $\psi_{+}\left(-L, x^{-}\right)=\psi\left(x^{-}\right)$and $\psi_{-}\left(x^{+},-L\right)=\widetilde{\psi}\left(x^{+}\right)$, where

$$
\begin{aligned}
& \psi\left(x^{-}\right)=\frac{1}{\sqrt{2 L}} \sum_{n=1,3, \ldots}^{\infty}\left(b_{n} e^{-\frac{i}{2} k_{n}^{+} x^{-}}+d_{n}^{\dagger} e^{\frac{i}{2} k_{n}^{+} x^{-}}\right) \\
& \widetilde{\psi}\left(x^{+}\right)=\frac{1}{\sqrt{2 L}} \sum_{n=1,3, \ldots}^{\infty}\left(\beta_{n} e^{-\frac{i}{2} k_{n}^{-} x^{+}}+\delta_{n}^{\dagger} e^{\frac{i}{2} k_{n}^{-} x^{+}}\right),
\end{aligned}
$$

and $k_{n}^{ \pm}=n \pi / L$. The correct commutation relations are

$$
\begin{aligned}
& \left\{\psi\left(x^{-}\right), \psi^{\dagger}\left(y^{-}\right)\right\}=\delta\left(x^{-}-y^{-}\right) \\
& \left\{\widetilde{\psi}\left(x^{+}\right), \widetilde{\psi}^{\dagger}\left(y^{+}\right)\right\}=\delta\left(x^{+}-y^{+}\right),
\end{aligned}
$$

with all other anticommutators vanishing, which correspond to

$$
\left\{b_{n}, b_{m}^{\dagger}\right\}=\left\{d_{n}, d_{m}^{\dagger}\right\}=\left\{\beta_{n}, \beta_{m}^{\dagger}\right\}=\left\{\delta_{n}, \delta_{m}^{\dagger}\right\}=\delta_{n, m}
$$

The Poincaré generators receive contributions from both wings of the initial-value surface [see Eqn. (3.16)], and correctly translate all fields in $x^{ \pm}$. Note that if the degrees of freedom represented by $\beta_{n}$ and $\delta_{n}$ are not included, then the theory contains only right-moving particles and is not equivalent to the equal-time theory of a massless Dirac fermion.

There are various ways of arriving at a correct construction for the case $m \neq 0$. Here I shall simply motivate one possible way, and then show that it works.

Let us start out by trying the same procedure as in the massless case. That is, we choose $\psi_{+}$to be an antiperiodic function $\psi\left(x^{-}\right)$on $x^{+}=-L$ and $\psi_{-}$to be an antiperiodic function $\widetilde{\psi}\left(x^{+}\right)$on $x^{-}=-L$. We shall also attempt to preserve the simple commutation relations (4.12). Now the Dirac equation allows us to obtain $\psi_{-}$on the surface $x^{+}=-L$ :

$$
\begin{aligned}
\psi_{-}\left(-L, x^{-}\right) & =\psi_{-}(-L,-L)-\frac{i m}{2} \int_{-L}^{x^{-}} d y^{-} \psi_{+}\left(-L, y^{-}\right) \\
& =\widetilde{\psi}(-L)-\frac{i m}{2} \int_{-L}^{x^{-}} d y^{-} \psi\left(y^{-}\right)
\end{aligned}
$$

(the treatment of $\psi_{+}\left(x^{+},-L\right)$ is completely symmetric, of course). It is helpful to rewrite this in the form

$$
\psi_{-}\left(-L, x^{-}\right)=\widetilde{\psi}(-L)-\frac{i m}{4} \int_{-L}^{L} d y^{-} \epsilon\left(x^{-}-y^{-}\right) \psi\left(y^{-}\right)-\frac{i m}{4} \int_{-L}^{L} d y^{-} \psi\left(y^{-}\right),
$$

from which we see that the simple commutation relations 4.10)-(4.11) do not correctly reproduce the anticommutator (4.6). The second term on the RHS of Eqn. (4.15) gives us what we want; the last term is the troublemaker. Let us attempt to fix this by defining $\psi_{-}\left(-L, x^{-}\right)$to be

$$
\psi_{-}\left(-L, x^{-}\right)=\widetilde{\psi}(-L)-\frac{i m}{4} \int_{-L}^{L} d y^{-} \epsilon\left(x^{-}-y^{-}\right) \psi\left(y^{-}\right)
$$


which gives the right commutator but changes the values of $\psi_{-}$at the endpoints. We now have

$$
\psi_{-}(-L, \pm L)=\widetilde{\psi}(-L) \mp \frac{i m}{4} \int_{-L}^{L} d y^{-} \psi\left(y^{-}\right)
$$

Note that equal-time antiperiodicity then implies

$$
\begin{aligned}
\psi_{-}(L,-L) & =-\psi_{-}(-L, L) \\
& =\widetilde{\psi}(L)+\frac{i m}{4} \int_{-L}^{L} d y^{-} \psi\left(y^{-}\right),
\end{aligned}
$$

suggesting that we should also modify the value of $\psi_{-}$at the point $(L,-L)$.

We are thus led to seek an expression for $\psi_{-}\left(x^{+},-L\right)$ that is more complicated than a simple antiperiodic function $\widetilde{\psi}$. Furthermore, the modified $\psi_{-}\left(x^{+},-L\right)$ should involve $\psi$, that is, should depend on $\psi_{+}\left(-L, x^{-}\right)$. A clue as to how to achieve this is obtained by thinking about causality. Recall that $\psi_{-}\left(x^{+},-L\right)$ and $\psi_{+}\left(-L, x^{-}\right)$should anticommute for $x^{+} \neq-L$ and $x^{-} \neq-L$. It would therefore be dangerous to include in $\psi_{-}\left(x^{+},-L\right)$ anything involving $\psi$ except at the endpoints $x^{+}= \pm L$; anything else would be likely to cause $\psi_{-}$ and $\psi_{+}$to fail to anticommute at space-like separation. This suggests a modification of $\psi_{-}\left(x^{+},-L\right)$ at the endpoints only, of the particular form

$$
\psi_{-}\left(x^{+},-L\right)=\widetilde{\psi}\left(x^{+}\right)+\frac{i m}{4}\left[2+\epsilon\left(x^{+}-L\right)-\epsilon\left(x^{+}+L\right)\right] \int_{-L}^{L} d y^{-} \psi\left(y^{+}\right) .
$$

That is, we add in a discontinuity at each endpoint designed to reproduce Eqns. (4.17)(4.18). This leaves the fields properly causal; away from the corner points $\psi_{-}\left(x^{+},-L\right)=$ $\psi\left(x^{+}\right)$and $\psi_{+}\left(-L, x^{-}\right)=\psi\left(x^{-}\right)$, which anticommute. Of course, the situation is symmetric with respect to $+\leftrightarrow-$ so that we should also take

$$
\psi_{+}\left(-L, x^{-}\right)=\psi\left(x^{-}\right)+\frac{i m}{4}\left[2+\epsilon\left(x^{-}-L\right)-\epsilon\left(x^{-}+L\right)\right] \int_{-L}^{L} d y^{+} \widetilde{\psi}\left(y^{+}\right) .
$$

Eqns. (4.19) and (4.20) are the final expressions for the independent fields on their respective initial-value surfaces.

As mentioned previously, there are other ways of arriving at these, but this one highlights the physical motivations behind the construction. Note that the fields are not strictly antiperiodic on the initial surfaces. That this must be the case follows immediately from integrating the equation of motion and imposing equal-time antiperiodicity; we obtain, e.g.,

$$
\psi_{-}(L,-L)+\psi_{-}(-L,-L)=\frac{i m}{2} \int_{-L}^{L} d x^{-} \psi_{+}\left(-L, x^{-}\right) .
$$

We have constructed the fields to be as antiperiodic as possible, however, while preserving the simple anticommutation relations (4.10)-(4.11). The motivation for this is that ultimately we would like to use this construction to obtain an effective Hamiltonian for the usual lightcone degrees of freedom $\left(\psi_{+}\right)$. We would therefore like the formalism to resemble as much as possible the usual one, with an antiperiodic $\psi_{+}$. We could perhaps have arrived at simpler expressions for the fields had we not insisted on maintaining simple Fock space commutation relations. 
The energy-momentum tensor has components

$$
\begin{gathered}
T^{+-}=T^{-+}=m\left(\psi_{-}^{\dagger} \psi_{+}+\psi_{+}^{\dagger} \psi_{-}\right) \\
T^{++}=2 i \psi_{+}^{\dagger} \partial_{-} \psi_{+}+\text {H.c. } \\
T^{--}=2 i \psi_{-}^{\dagger} \partial_{+} \psi_{-}+\text {H.c. }
\end{gathered}
$$

and the Poincaré generators work out to be [Eqn. (3.16)]

$$
\begin{gathered}
P^{-}=-\frac{i m^{2}}{4} \int_{-L}^{L} d x^{-} \int_{-L}^{L} d y^{-} \psi^{\dagger}\left(x^{-}\right) \epsilon\left(x^{-}-y^{-}\right) \psi\left(y^{-}\right)+2 i \int_{-L}^{L} d x^{+} \widetilde{\psi}^{\dagger}\left(x^{+}\right) \partial_{+} \widetilde{\psi}\left(x^{+}\right) \\
+m \widetilde{\psi}^{\dagger}(-L) \int_{-L}^{L} d x^{-} \psi\left(x^{-}\right)+m\left[\int_{-L}^{L} d x^{-} \psi^{\dagger}\left(x^{-}\right)\right] \widetilde{\psi}(-L) \\
P^{+}=-\frac{i m^{2}}{4} \int_{-L}^{L} d x^{+} \int_{-L}^{L} d y^{+} \widetilde{\psi}^{\dagger}\left(x^{+}\right) \epsilon\left(x^{+}-y^{+}\right) \widetilde{\psi}\left(y^{+}\right)+2 i \int_{-L}^{L} d x^{-} \psi^{\dagger}\left(x^{-}\right) \partial_{-} \psi\left(x^{-}\right) \\
+m \psi^{\dagger}(-L) \int_{-L}^{L} d x^{+} \widetilde{\psi}\left(x^{+}\right)+m\left[\int_{-L}^{L} d x^{+} \widetilde{\psi}^{\dagger}\left(x^{+}\right)\right] \psi(-L) .
\end{gathered}
$$

These can be shown to correctly translate all fields in $x^{ \pm}$, including the fields at the corner points, and furthermore satisfy $\left[P^{+}, P^{-}\right]=0$. We thus have a satisfactory representation of the theory with the boundary degrees of freedom present. Note the presence of terms coupling together the fields on the two wings.

A few technical points are worth noting. First, the discontinuities in the fields at the corner points lead to singularities in their derivatives, and these give rise to important contributions in the pieces of $P^{\mu}$ involving $T^{++}$and $T^{--}$. Second, in verifying the Heisenberg relations or the Poincaré algebra, it should be kept in mind that since $\psi\left(x^{-}\right)$is antiperiodic, it follows that, for example,

$$
\left\{\psi\left(x^{-}\right), \psi^{\dagger}(-L)\right\}=\delta\left(x^{-}+L\right)-\delta\left(x^{-}-L\right) .
$$

Furthermore, when the argument of a delta function vanishes at an endpoint of the integration region, the proper definition of the integral is, e.g.,

$$
\int_{-L}^{L} d x^{-} f\left(x^{-}\right) \delta\left(x^{-}-L\right)=\frac{1}{2} f(L) .
$$

It is now fairly simple to obtain suitable expressions for the fields for use with the asymmetric initial-value surface shown in Fig. 2(b). These may be obtained from Eqns. $(4.19)$ and $(4.20)$ by a shift of $x^{+}$and a slight reorganization of the resulting expressions [11]. For the independent fields we obtain

$$
\psi_{+}\left(0, x^{-}\right)=\psi\left(x^{-}\right)+\frac{i m}{4}\left[2+\epsilon\left(x^{-}-L\right)-\epsilon\left(x^{-}+L\right)\right] \int_{-L}^{L} d y^{+} \epsilon\left(y^{+}\right) \widetilde{\psi}\left(y^{+}\right)
$$


and

$$
\psi_{-}\left(x^{+}, \pm L\right)=\widetilde{\psi}\left(x^{+}\right)-\frac{i m}{4}\left[\epsilon\left(x^{+}\right) \pm 1\right] \int_{-L}^{L} d y^{-} \psi\left(y^{+}\right)
$$

Integrating the equation of motion then gives us the constrained fields:

$$
\begin{gathered}
\psi_{+}\left(x^{+}, \pm L\right)=\psi( \pm L)-\frac{i m}{4} \int_{-L}^{L} d y^{+} \epsilon\left(x^{+}-y^{+}\right) \widetilde{\psi}\left(y^{+}\right) \\
\psi_{-}\left(0, x^{-}\right)=\widetilde{\psi}(0)-\frac{i m}{4} \int_{-L}^{L} d y^{-} \epsilon\left(x^{-}-y^{-}\right) \psi\left(y^{-}\right) .
\end{gathered}
$$

The Poincaré generators are now obtained by integrating

$$
P^{ \pm}=\frac{1}{2} \int_{-L}^{0} d x^{+} T^{- \pm}\left(x^{+}, L\right)+\frac{1}{2} \int_{-L}^{L} d x^{-} T^{+ \pm}\left(0, x^{-}\right)+\frac{1}{2} \int_{0}^{L} d x^{+} T^{- \pm}\left(x^{+},-L\right) .
$$

We find

$$
\begin{gathered}
P^{+}=-\frac{i m^{2}}{4} \int_{-L}^{L} d x^{+} \int_{-L}^{L} d y^{+} \widetilde{\psi}^{\dagger}\left(x^{+}\right) \epsilon\left(x^{+}-y^{+}\right) \widetilde{\psi}\left(y^{+}\right)+2 i \int_{-L}^{L} d x^{-} \psi^{\dagger}\left(x^{-}\right) \partial_{-} \psi\left(x^{-}\right) \\
+m \psi^{\dagger}(-L) \int_{-L}^{L} d x^{+} \epsilon\left(x^{+}\right) \widetilde{\psi}\left(x^{+}\right)+m\left[\int_{-L}^{L} d x^{+} \epsilon\left(x^{+}\right) \widetilde{\psi}^{\dagger}\left(x^{+}\right)\right] \psi(-L) \\
P^{-}=-\frac{i m^{2}}{4} \int_{-L}^{L} d x^{-} \int_{-L}^{L} d y^{-} \psi^{\dagger}\left(x^{-}\right) \epsilon\left(x^{-}-y^{-}\right) \psi\left(y^{-}\right)+2 i \int_{-L}^{L} d x^{+} \widetilde{\psi}^{\dagger}\left(x^{+}\right) \partial_{+} \widetilde{\psi}\left(x^{+}\right) \\
+m \widetilde{\psi}^{\dagger}(0) \int_{-L}^{L} d x^{-} \psi\left(x^{-}\right)+m\left[\int_{-L}^{L} d x^{-} \psi^{\dagger}\left(x^{-}\right)\right] \widetilde{\psi}(0)
\end{gathered}
$$

Again, these can be shown to properly translate the fields and to commute with each other.

\section{DISCUSSION}

I have argued that in a finite volume, introduced as an infrared regulator, including degrees of freedom not present in the usual light-cone formalism is necessary if the resulting theory is to be equivalent to the corresponding equal-time theory. This follows from analyzing the equations of motion, as well as from constructing Poincaré generators and other charges that are the same as the operators we would construct in equal-time quantization. I have also attempted to give some flavor of the formalism necessary to achieve this equivalence. A major advantage of this approach is that it gives a regulated theory but does not force the physical vacuum to be trivial. This is a welcome feature, as the QCD vacuum, for example, is certainly not trivial.

Its main disadvantage is that it is quite complicated, even at the level of free field theory. In fact, it is more complicated than equal-time field theory, so that if the point were to use it directly as a calculational tool it would be largely useless. However, recall that vacuum 


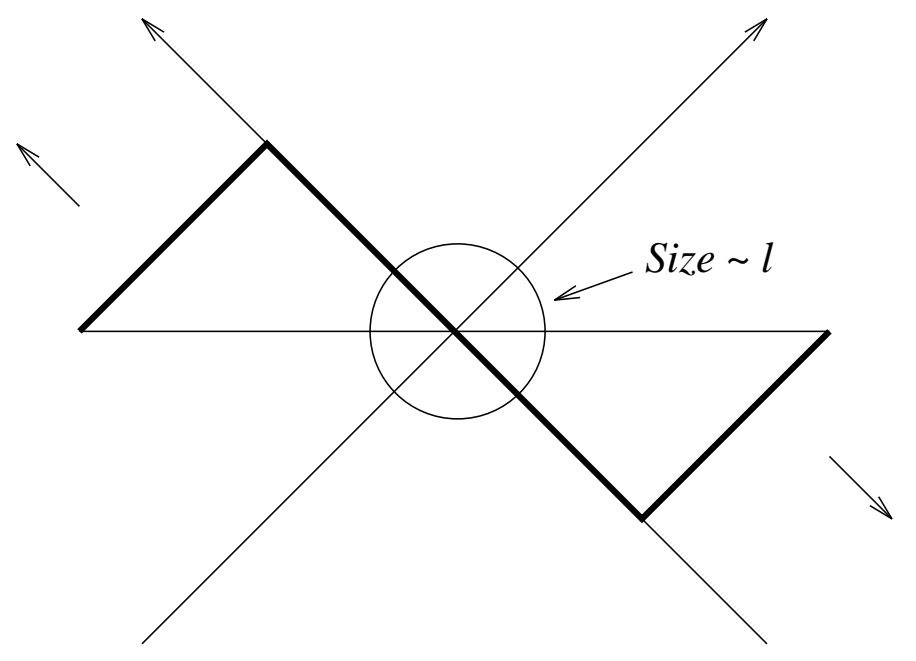

FIG. 4. Decoupling the boundaries in the large- $L$ limit.

triviality on the light cone really means that the effects of the vacuum should be expressible in terms of local effective interactions for a set of fields initialized at a fixed $x^{+}$. That is, we should be able to construct an effective theory which has a trivial vacuum state, but nontrivial vacuum physics. The light-cone framework is the only one in which such an approach is possible, due to the unusual connection between long distances (in $x^{-}$) and high (light-cone) energies reflected in the dispersion relation (1.1).

This construction can be used to obtain such effective Hamiltonians in a natural way. The basic idea is to consider the limit in which the box size $L$ becomes large compared to some fixed scale $l$ of physical interest [Fig. 4]. In this case, the fields that live on the boundaries appear to decouple from those that live at $x^{+}=0$; by causality, quanta from the boundaries that can influence the region of interest have their world lines pushed closer and closer to the light cone as the box gets large. This decoupling may be illusory, however, because the couplings of these states can simultaneously diverge. The goal is to extract the finite remainders in this limit, and build them in to an effective Hamiltonian for use an a "traditional" light-cone calculation. Details of some simple examples are given in [11].

This formulation of the light-cone theory with boundaries is also instructive from the point of view of the puzzles listed in Sect. I. For example, in the language of partial differential equations the construction corresponds to the "characteristic" initial-value problem, as distinct from the Cauchy problem. This is perfectly well defined from a mathematical point of view. There is no real problem with using surfaces of characteristics to specify initial conditions, but more than one such surface is necessary in general.

The question of what boundary conditions may be imposed on the characteristic surfaces can be addressed naturally in the course of setting up a concrete realization of the scheme. In general, imposing strict periodicity or antiperiodicity is seen to be in conflict with the dynamics, and results in the removal of degrees of freedom.

The massless limit of the construction is perfectly smooth; the degrees of freedom that seem to appear only for $m=0$ are actually necessary for the massive theory as well, and so are present from the outset. As discussed above, the confusion over whether the extra fields are necessary in the massive case arises from considering the artificially simple case of free theory, where the boundaries do decouple for massive fields. In general they are required, 
and when they are present there is nothing discontinuous, or otherwise unusual, about the massless limit.

Regarding the "No-problem" problem, the construction allows us to study directly the effects of discarding various pieces of physics. It would be very helpful to have a quantitative understanding, for example, of what could be computed reliably without including the boundaries. The present scheme allows this to be studied concretely. There are also more specific questions along these lines that can be considered. For example, what is the relation between the effective interactions induced by the constrained zero modes in DLCQ and the effective interactions one obtains in the full theory? Do they in fact capture some sort of "mean field" vacuum properties, and if so, what can be calculated reliably in that framework without addressing the full boundary problem?

It is perhaps worth restating that the potential advantage of light-cone quantization over equal-time quantization is not that the vacuum becomes trivial, but rather that the vacuum can trivially be made trivial, by means of a simple cutoff on longitudinal momenta. This has several immediate advantages. When coupled with the insight that the states thus removed are mainly high-energy states in $3+1$ dimensions, it leads to the hope that we might be able to derive a constituent approximation to QCD. In fact, it is the only framework that offers any realistic hope of achieving this. In addition, for problems where the vacuum does not play an important physical role the ability to ignore it so simply may be quite useful. In QCD, for example, it is presumably not necessary to completely understand the pion or to have strictly linear long-range potentials in order to obtain a reasonable description of, e.g., heavy quarkonia. For this sort of problem it may be quite reasonable to ignore the effective interactions that represent the vacuum, or, equivalently, to use the conventional light-cone formalism without boundary fields.

For problems where the vacuum does play a role, however, its structure must be properly accounted for. The formalism described here, or something equivalent, will be necessary to obtain the effective interactions that mediate vacuum physics. For QCD this can be expected to be quite difficult, in part because the problem is intrinsically nonperturbative. Of course, the QCD vacuum is a difficult problem in any (known) calculational scheme! In any case, the possibility of obtaining a constituent approximation to QCD, complementary to the lattice, and of making contact with the natural and very successful phenomenology based on the light-cone Fock representation, makes this set of problems of considerable interest.

\section{ACKNOWLEDGMENTS}

It is a pleasure to thank the organizers of ORBIS SCIENTIAE 1996 for providing such a stimulating and enjoyable atmosphere. This work was done in collaboration with Kent Hornbostel, and I am grateful to him for many enjoyable and illuminating discussions on the subject of light-cone field theory. I also thank Gary McCartor and Robert Perry for many helpful conversations regarding the light-cone vacuum. This work was supported in part by a grant from the the U.S. Department of Energy. 


\section{REFERENCES}

[1] For an overview of QCD phenomenology from the light-cone point of view, see: S. J. Brodsky, these proceedings; S. J. Brodsky and D. G. Robertson, "Light-Cone Quantization and QCD Phenomenology," hep-ph/9511374, to appear in the proceedings of the ELFE Summer School and Workshop on Confinement Physics, Cambridge, UK, July 1995.

[2] K. Hornbostel, Phys. Rev. D 45, 3781 (1992).

[3] R. J. Perry, Ann. Phys. 232, 116 (1994); K. G. Wilson, T. S. Walhout, A. Harindranath, W.-M. Zhang, R. J. Perry, and St. D. Glazek, Phys. Rev. D 49, 6720 (1994).

[4] S. J. Brodsky and H.-C. Pauli, in Recent Aspects of Quantum Fields, H. Mitter and H. Gausterer, Eds., Lecture Notes in Physics, Vol. 396 (Springer-Verlag, 1991), and references therein.

[5] T. Heinzl, S. Krusche, S. Simbürger, and E. Werner, Z. Phys. C 56, 415 (1992); D. G. Robertson, Phys. Rev. D 47, 2549 (1993); C. M. Bender, S. S. Pinsky, and B. van de Sande, Phys. Rev. D 48, 816 (1993); S. S. Pinsky and B. van de Sande, Phys. Rev. D 49, 2001 (1994); J. Hiller, S. S. Pinsky, and B. van de Sande, Phys. Rev. D 51, 726 (1995).

[6] F. Rohrlich, Acta Phys. Austr. 32, 87 (1970); P. J. Steinhardt, Ann. Phys. 128, 425 (1980).

[7] G. McCartor, Z. Phys. C 41, 271 (1988).

[8] H. Bergknoff, Nucl. Phys. B 122, 215 (1977); T. Eller, H.-C. Pauli, and S. J. Brodsky, Phys. Rev. D 35, 1493 (1987).

[9] G. McCartor, Z. Phys. C 52, 611 (1991); ibid. 64, 349 (1994).

[10] K. Hornbostel, Ph. D. thesis, Stanford University (1988).

[11] K. Hornbostel and D. G. Robertson, manuscript in preparation (1996). 